\title{
Design and Numerical Simulation of Axial-flow Wind Turbine
}

\author{
Haowei Hu \\ Department of Energy Power and Mechanical Engineering, North China Electric Power University, \\ Baoding, 071003, China
}

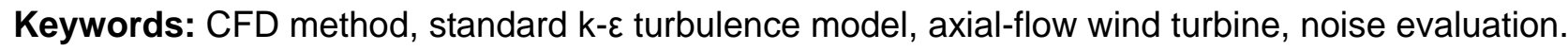

\begin{abstract}
With the development of the modern society, new energy technology has a great potential to be the new driving force. In order to make full use of the wind energy in the high air, based on CFD method, using the commercial software FLUENT and the standard k- $\varepsilon$ turbulence model, an axial-flow wind turbine was designed on its structure and simulated with its inner flow field, also the pressure and velocity contours were obtained and the noise evaluation was considered. The results show that for the axial-flow wind turbine, setting the appropriate blade installation angle can be helpful for blade to bear balanced pressure and for air to flow steadly, which offers references to reduce noise and prolong the life of the blade in the industral application.
\end{abstract}

\section{Introduction}

In recent years, as a clean and renewable energy, wind-power resource gains an increasing number of attention all over the world. In 1888, Charles F. Brush designed the first wind turbine as the symbol that wind turbine came to reality for the first time ${ }^{[1]}$. With the deepening of the study, through wind tunnel tests, the rotation and oscillation characteristics of airflow in a turbulent state were studied by C.Sicot, P.Devimnt et al, providing more precise methods for analysis of blade's aerodynamic characteristics ${ }^{[2]}$. But wind direction has uncertainty, regionalism and intermittent, which makes people gradually put forward using high-altitude wind resource with wind turbine in the high air $^{[3]}$. However, the technology is limited by the following factors: first of all, because of the intermittent problem of the wind, the energy of collected wind-power is extremely unstable; secondly, owing to the existence of low-frequency noise, the scale of deployment was limited; finally, the blade currently used is traditional fan leaf type. When sent to the high air, it's easy to damage the generator by heavy wind and greatly reduce the efficiency of the machine.

Out of the above consideration, for using the wind energy efficientively and balancing the pressure exerted on the blade, this artical proposes an axial-flow wind turbine which can be used in high air to improve the blade structure, enhance the power generation efficiency, prolong the life of blade and reduce noise.

\section{Basic theory of flow ${ }^{[4-6]}$ \\ Velocity triangle}

When Calculating axial-flow of the fluid, it's general to think that the fuid micelles within the field is flowing in the axis line of the cylindrical surface, and the fluids in two adjacent cylindrical surface behaves as disparate flows, that is, there's no radial velocity in each move. Note the absolute velocity, convected velocity and relative velocity respectively as $v, u, \omega$, then:

$$
\vec{v}=\vec{u}+\vec{\omega}
$$

By fluid's continuity and incompressibility, it's knowable that in the same radius of blade the convected velocities are equal, namely, $u_{1}=u_{2}=u$, also the blade's axial-direction of relative velocity and absolute velocity are identical, i.e. $\omega_{1 \mathrm{a}}=\omega_{2 \mathrm{a}}=\omega_{\mathrm{a}}, v_{1 \mathrm{a}}=v_{2 \mathrm{a}}=v_{\mathrm{a}}$. For axial flow, the velocity triangle is shown below: 


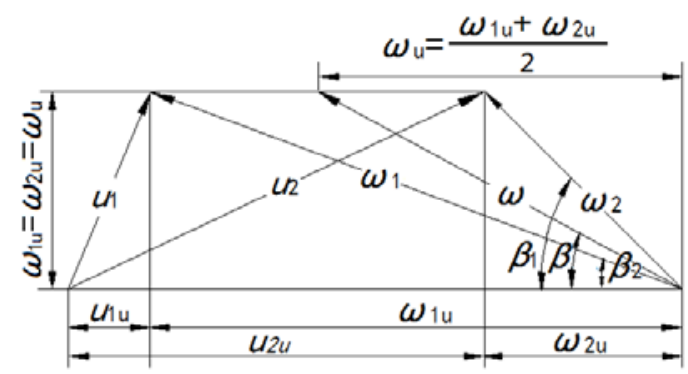

Fig.1: velocity triangle

Because the blade changes the flow direction, if we use the average of $\omega_{1}, \omega_{2}$ to replace the infinity flow velocity $\omega$, then the installation angle can be presented as:

$$
\tan \beta=\frac{\omega_{a}}{\omega_{u}}=\frac{2 \omega_{a}}{\omega_{1 u}+\omega_{2 u}}
$$

\section{Wing theory}

The airfoil is the geometric line which has a certain aerodynamic characteristics. In 1909, Zhukovskiy firstly proposed the theory of incompressible fluid flow around airfoil, obtaining the formula as follows:

$$
\Gamma=\pi u b \sin \left(\alpha-\alpha_{0}\right)
$$

among them, $b$ stands for airfoil chord length, $\alpha$ is the angle of attack and $\alpha_{0}$ is the zero-incidence . The lift formula is concluded as:

$$
F_{L}=\rho u \Gamma
$$

the flow-direction rotates against vortex by $\pi / 2$.

\section{Set of model}

\section{Physical model}

The set of the axial-flow wind turbine was shown in Figure 2(a). The hub diameter $d=200 \mathrm{~mm}$, casing inner diameter $D=320 \mathrm{~mm}$, leaf height $h=50 \mathrm{~mm}$, tip clearance $\delta=10 \mathrm{~mm}$, blade number $z=6$, axial-length $L=250 \mathrm{~mm}$. In order to make the blade windward area bear balanced pressure, blade installation angle $\beta$ is set as $30^{\circ}$.

The simulated object for axial-flow wind turbine was its inner flow field. In order to make full use of wind resources in the sky, the device is flied to the altitude of 300 meters, so the corresponding inlet pressure is $97.33 \mathrm{kPa}$ and wind speed is approximate to $12 \mathrm{~m} / \mathrm{s}$. Because of the installation of the speed-limit device, the hub’s absolute speed is districted below $1200 \mathrm{r} / \mathrm{min}$.

Considering the geometric-symmetry of the model, the numerical flow field is taken only 1/6 of practical flow field, as Fig. 2 (b) shows.

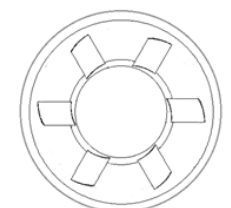

(a)geometric model

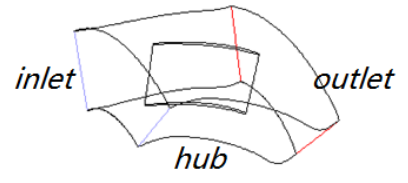

(b)flow field

Fig. 2: model set

\section{Mathematical model}

Considering the flow state is turbulent flow, the standard $k-\varepsilon$ turbulence model is choosen to solve. The standard $k-\varepsilon$ equation using the turbulent kinetic energy $k$ and turbulence dissipation rate $\varepsilon$ to present the turbulent viscosity $\mu_{\mathrm{t}}$. 


$$
\begin{gathered}
k=\frac{1}{2} \overline{v_{i}^{\prime} v_{j}^{\prime}} \\
\varepsilon=v \overline{\left(\frac{\partial v_{i}^{\prime}}{\partial x_{k}}\right)\left(\frac{\partial v_{j}^{\prime}}{\partial x_{k}}\right)} \\
\mu_{t}=\rho C_{\mu} \frac{k^{2}}{\varepsilon}
\end{gathered}
$$

$v^{\prime}$ is the mean velocity, $v$ is the kinematic viscosity and $C_{\mu}$ is a empirical constant.

\section{Results and analysis}

\section{Simulation results}

Drawing geometric model in GAMBIT and dividing the mesh, the total numbers of mesh is 209646 and the quality are all between $0 \sim 1{ }^{[7]}$. After calculating in the FLUENT software, we can get the following simulation results.

Fig. 3 and Fig. 4 represent pressure distribution on the blade, hub surface and suction surface respectively. As can be seen from the graph, the maximal pressure comes near the blade leading edge, then decreases with the airflow moving, and the pressure of the hub surface is greater than that of the suction surface. As the leading edge is the stationary point of the airflow, where the flowstate changes significantly, the airflow will exert a strong attack on the blade. And it can be seen from the figure that the pressure's change is slow and slight, so the machine only suffers small impact-force and has certain life span.
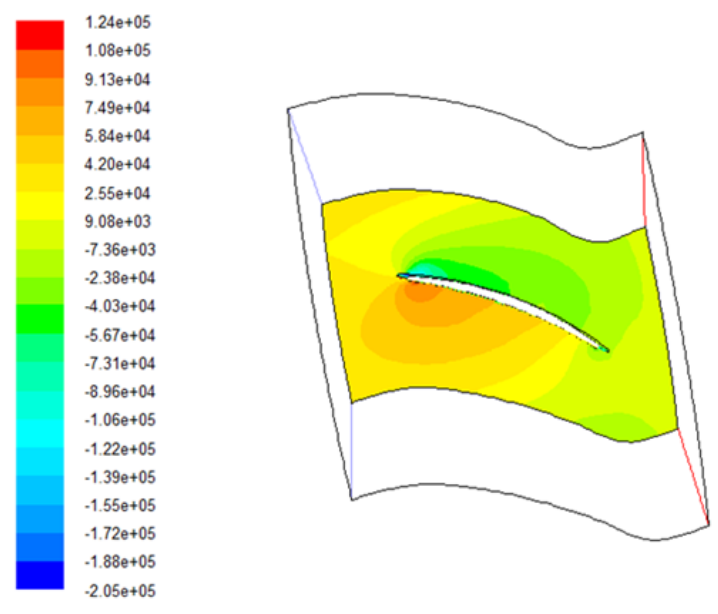

Fig. 3: Schematic diagram of force distribution of the hub

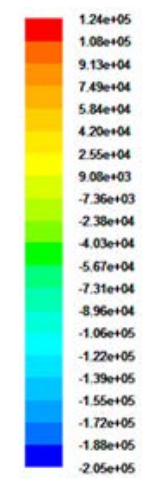

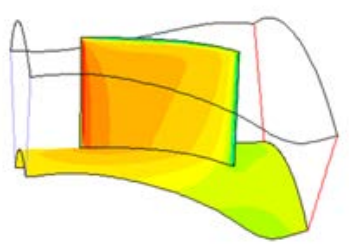

pressure

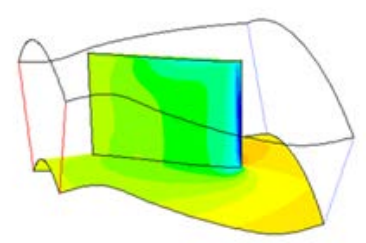

suction

Fig. 4: Schematic diagram of force distribution of the pressure and suction surface 
Fig. 5 is the velocity contour in the flow field. As can be seen from the graph, near the leading and trailing edge the airflow encounters blockade by blades heavily, while in the tip clearance the velocity is fluent. When the blade installation angle is $30^{\circ}$, while the fluid's velocity changes in flow process, this change-amplitude is small enough so that it can ensure the safety and reliability of machine in high-altitude operation to the certain extent.
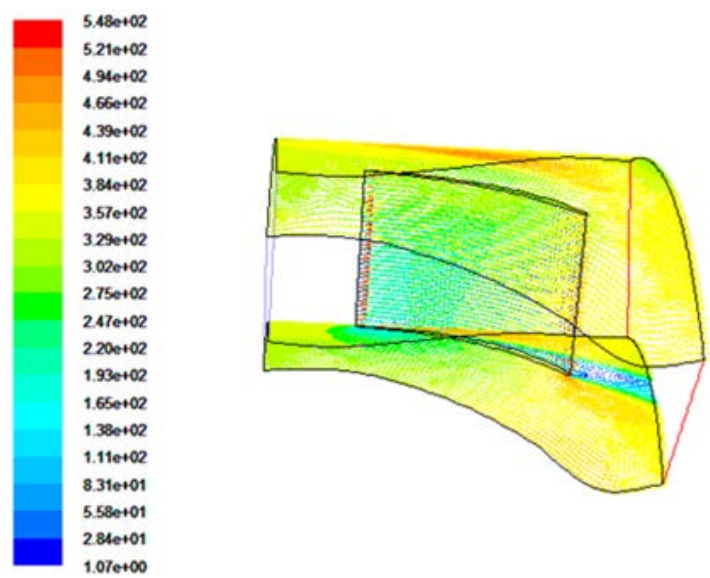

Fig. 5: Schematic diagram of velocity distribution

Fig.6 shows the distribution of streamline in the inner flow passage. On account that the streamline can reflect roughly the trajectory of airflow movement from the side, as can be seen from the graph, in every section of the flow field, the streamline's distribution is uniform and the streamline is smooth, also the extent of bending is slight and no obvious inflection point exists, so the air flow can be considered stable, and there's no significant impact of the air flow in the whole flow field, which is of great help to improve the use of generator.
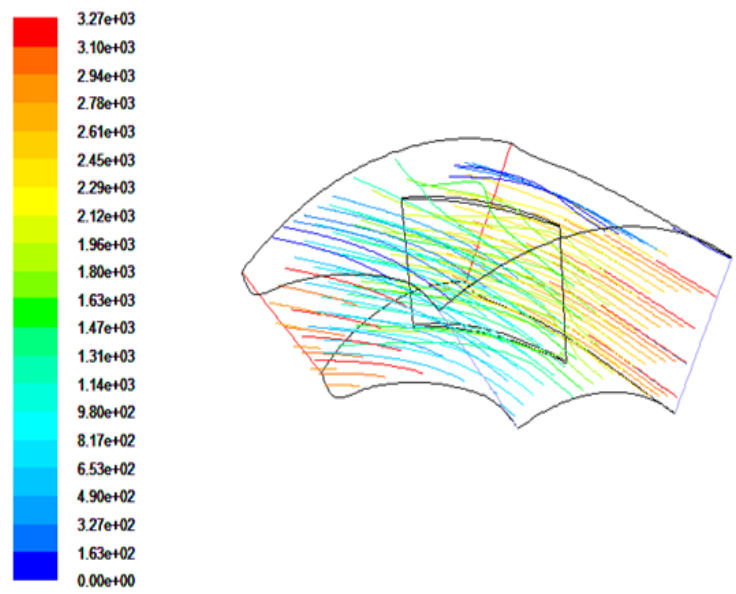

Fig. 6: Schematic diagram of streamline

Figure 7 shows the variation of static pressure from the hub to the casing. For the rotating blade part, in different position the impact of airflow is various, and the further it leaves from hub, the bigger the bending moment will be exerted, so the static pressure with distance away from the hub is a linear growth regularity; and in tip clearance region, because the flow of the air is not restricted, and airflow rate is stable, so the static pressure drops sharply. To sum up,in the whole inner field, because the static pressure distribution is uniform, so the blade design is appropriate. 


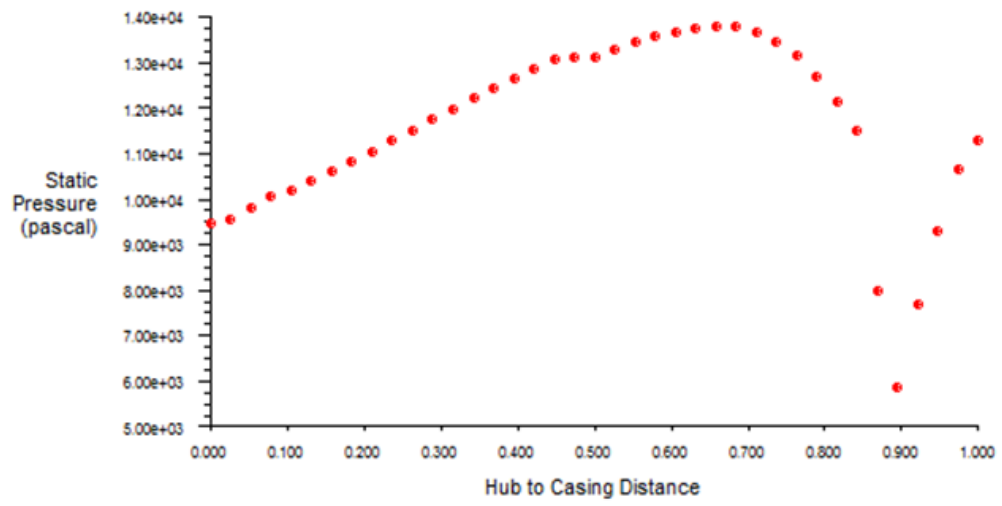

Fig. 7: Schematic diagram of pressure from hub to casing

\section{Noise evaluation}

For further, it's necessary to make the noise evaluation ${ }^{[8]}$. The emergence of the noise is mainly based on the following factors: when the blade is rotated by the periodic airflow shocks it will exert a pulsating wind pressure; at the same time the boundary layer is formed and then falls in the trailing edge of the blades, which caused the wind pressure intensify. The noise is associated with the hub's speed, blade number, blade geometry and so on, and from the simulation results we can see that when the blade is designed into the axial-flow type, the appropriate installation angle can make the velocity of airflow considerably stable and sharpen the tail flow separation area, so the noise can be minimized and ensure the certain security.

\section{Conclusions}

In this artical, based on CFD, by numerical simulation, we can obtaine the following conclusions:

(1) Wind-power in high air has a broad prospect, which can be used in aerospace, meteorology and other areas of investigation.

(2) Compared wih traditional fan blades, axial-flow wind turbine owes a smoother airflow to extend life span of the blades; at the same time, selecting the appropriate installation angle can make the blade bear smaller wind-attack. What's more, the design can effectively reduce the blades' rotation noise, ensuring its stable operation in high air.

(3) The CFD method and the FLUENT software play an important role in designing and selecting the appropriate fan. By using them, we can proceed reasonable design for the strucure of some induetrial machine.

\section{Acknowledgements}

The research work was supported by National Natural Science Foundation of China under Grant No. 61065009 and Natural Science Foundation of Qinghai Provincial under Grant No. 2011-z-756.

\section{References}

[1]Zuo Ran, Shi Mingheng, Tuo Xilin. Introduction of Renewable Energy[M]. Beijing:Mechanical Industry Press,2007:1-30.

[2]C.Sicot, P.Devinant, S.Loyer, et al. Rotational and turbulence effects on a wind turbine blade. Investigation of the stall mechanisms [J]. Journal of Wind Engineering and Industrial Aerodynamics, 2008, 98: 1320-1331.

[3]Ho R H S.Lateral Stability and Control of a Flying Wind Generator[D]. Sydney:University of Sydney,1992.

[4]Irving Granet. Fluid Mechanics for Engineering Technology[M]. Prentice-Hall Inc,1981. 
[5]Wang Songling. Fluid Mechanics [M]. Beijing: China Electric Power Press, 2007: 217-225.

[6]An Liansuo, Lv Yukun. Fluid Machinery[M].Beijing: China Electric Power Press,2013:13-15.

[7]Han Zhanzhong, Wang Jing, LAN Xiaoping. FLUENT Fluid Engineering Simulation Calculation Example and its Application[M]. Beijing: Beijing Institute of Technology press, 2004.

[8]Yu Wenwen. A Dissertation Submitted in Partial of Fulfillment of the Requirements For the Degree of Master Engineering[D].Wuhan:Huazhong University,2007. 